\title{
MEDICAL AND POPULATION STATISTICS FOR ENGLAND AND WALES
}

\begin{abstract}
$\mathrm{T}$ HE two volumes of vital statistics published by the Registrar General for 1961 have recently become available*. There have been a number of changes in presentation : on the medical side causes of stillbirth have been tabulated for the first time; on the eivil side the new data available under the Population (Statisties) Act 1960 relating to the ages of fathers of children born are presented for all legitimate births, and for those illegitimate births $(37 \cdot 8$ per cent of the total) which are registered by the parents jointly. The year under review saw no very startling changes. Mortality was slightly higher than in 1960 , the crude death rate having risen by 0.4 for males and 0.5 for females to 12.6 and 11.4 respectively. The principal cause for this rise appears to be the large number of deaths from respiratory infection (mainly influenza and pneumonia) in the first quarter. The figures for cause mortality underline the decline in tuberculosis deaths, of which 3,334 were registered in 1961 against 12,031 ten years ago. Motor vehiclo accidents causod 6,544 deaths, 13 fower than in 1960, but still nearly 50 per cent more than ten years ago. 'The death rate from malignant neoplasms has remained steady over the past three years, but the distribution by sites is still changing, cancer of the respiratory system continuing to
\end{abstract}

* The Registrar General's Statistical Review of England and Wales for the Population. Pp. xili 189. 15s. net. (London: H.M.S.O., 1963.)

\section{LASERS, ULTRA-SONICS}

$\mathrm{T}$ HE texts of the three discourses delivered by Dr. J. M. Burch (National Physical Laboratory, Teddington), Dr. C. F. Brocklesby (Mullard Research Laboratories, Redhill), and Dr. J. A. Ratcliffe (Radio Research Station, Slough), respectively, at the annual exhibition of scientific instruments and apparatus of the Institute of Physics and the Physical Society during January 15-17, are printed in the April issue $(40,147 ; 1963)$ of the Journal of Scientific Instruments.

Dr. Burch, who spoke on the subject of optical research and the solid-state laser, dealt first with the various types of laser and the progress made during the past two years in both the search of new and better laser materials and the improvement in performance of the device. He outlined the possibilities of application of lasers in practical technology and scientific research and then turned to a discussion of the questions of how the optical maser works and how the device, invented by microwave physicists, may be described in optical and non-mathematical terms. Three of the main limitations to the optimum performance of solid-state lasers, at present, were, the divergence of the beam caused by variations in refractive index of the laser material, the frequency drift caused by the optical pump, and the 'spiking' or instability of tho light output. Dr. Burch briefly referred to recent work related to the elimination of these difficulties and concluded by saying that doubtless new advances would be reported at the third Quantum Electronics Conference to bo held in Paris during February 1963.

The second discourse on some applications of ultrasonics was given by Dr. Brocklesby. Outlining the history of ultra-sonics, he explained that the many applications in science and industry during the past thirty years consisted of two types-one based on power, where the purpose is to produce a mechanical, physical increase. Infant mortality is at a record low at 21 per 1,000 live births. The new stillbirth tabulations show that out of a total rate of 19 per 1,000 live births, 5.6 wero due to maternal conditions, $\mathbf{4 \cdot 9}$ to placental and cord conditions and 8.5 to foetal conditions. The number of births registered was the highest since 1922 with the exception of 1946 and 1947. In the five years 1956-61 the number of births rose from 700,000 to 811,000 , the live birth rate from $15 \cdot 6$ to $17 \cdot 5$ and the legitimate fertility from $108 \cdot 2$ to $122 \cdot 1$ per 1,000 married women aged 15-44. The proportion of illegitimate births rose from 5 per cent to 6 per cent of all births, but in 37.8 per cent the birth was registered by the father and mother jointly, suggesting that these might be births to couples living in stable unions, a suggestion reinforced by the fact that in 37 per cent of these cases the father was more than 35 years old, as against 23 per cent of the fathers of legitimate children.

Statistics of family size are difficult to interpret; comparing 1961 with 1956 , mean family size to women married for five years rose from 1.18 to 1.32 : at 10 years' duration, however, the rise was only from 1.80 to 1.82 : and at 20 years' duration the figures were practically constant at 1.99 and 2.00 . It is thus still feasible that the recent large number of births merely represent $\mathbf{a}$ shift in timing, rather than an increase in family size. The fertility tabulations of the 1961 consus should throw further light on this topic.

E. GREBENIK

or possibly a chemical change in some material, and the other aimed at obtaining information about the material rather than producing a modification of the material. The various applications cover a wide field, not only in industry and medicine, and in the scientific study of the properties and structures of materials, but also in some problems of communications and data handling. Focused ultra-sound is now used in surgery, and echo-sounding techniques have recently been utilized in medical diagnosis. In the fields of metallurgy and the processing of solids ultra-sonic welding and ultra-sonic drilling are well-established practices. In communications the most important application is that of the ultra-sonic delay line. The solid delay line has almost completely replaced the liquid delay line, and by using vitreous silica and the total internal reflexion which occurs at the glass/ air surface it is now possible to fold the path of the ultrasonic waves by repeated reflexions so that an equivalent long delay path can be obtained within a comparatively short length of silica. For delays of $100 \mu \mathrm{s}$, signal bandwidths of up to $40 \mathrm{Mc} / \mathrm{s}$ have been achieved and significant increases in bandwidth are in progress. A field of application which is related to delay lines is the interaction of light with ultra-sound in transparent media. For example, the stress-optical effect in glass can be used with ultra-sound to display the individual waves of the ultra-sound as fluctuations of brightness, or the variation in refractive index with stress of the transparent medium can be utilized to produce a high-speed light modulator, because the medium traversed by the ultra-sonic waves becomes a transmission diffraction grating.

"The Experimental Investigation of Space" was the title of Dr. Ratcliffe's talk. He discussed the experiments which have been and are being made, with the help of 
rockets and artificial satellites, to investigate phenomena above the Earth's surface. To get 'outside' the Earth's atmosphere to explore the external radiations space vehicles must go to heights greater than about $120 \mathrm{~km}$. Satellites cannot approach nearer than about $250 \mathrm{~km}$ if they are to remain long in orbit, and in order to get away from the ionized upper atmosphere we must use a space-probe which travels a distance of at least ten to fourteen Earth radii. With rocket-borne spectroscopes, ionization chambers and cameras, experiments have been made on observations of the ultra-violet rays and $\mathrm{X}$-rays of wave-lengths less than about $4000 \AA$ emitted from the Sun, and it has been established that the ultra-violet radiation comes only from localized areas of the Sun, and that the X-radiations of wave-lengths less than $10 \AA$ come from those active areas of the Sun which emit also radionoise of wave-length $10 \mathrm{~cm}$. As an example of what can be done with artificial satellites Dr. Ratcliffe discussed the measurement of the variation of electron concentration with height in the upper part of the Earth's atmo. sphere. The results obtained show that the electron concentration corresponds to an atmosphere the mean atomic mass of which decreases upwards, indicating that there is a gradual change from predominance in atomic oxygen at a height of about $300 \mathrm{~km}$ to predominance of helium at about $1,000 \mathrm{~km}$.

The detection of charged particles trapped in the Earth's magnetic field and the way in which these particles are trapped have been investigated by space probessatellites which travel around the Earth in very elliptical orbits but which never completely escape from the Earth's attraction. Magnetometers carried by these space probes have been used to measure the ring current, that is the sideways drift around the Earth of the positive and negative charges trapped in the Earth's magnetic field, and to investigate the magnetic field out to great distances from the Earth. It has been shown that the field decreases up to distances of from about ten to fourteen Earth radii but that beyond the decrease ceases and the field becomes irregular.

\section{OPTICAL MASERS}

C PHILLIPS remarks in his report (Journal of ScienJ. tific Instruments, 40, 89 ; March 1963) on the conference on optical masers held by the Institute of Physics and the Physical Society in London during September 1962, that since C. H. Townes's prediction two years ago the reaction to lasers has been exceedingly great in the United States, but comparatively only cautious in Great Britain. It is therefore not surprising, but significant, that only one of the twenty contributions to Supplement No. 1, Optical Masers, of Applied Optics*, is by a British author, but that this is the major and leading article.

The Supplement contains two long review articles and eighteen shorter papers, all dealing with various aspects of solid-state or gaseous optical masers. The first, by O. S. Heavens of the Royal Holloway College, University of London, concentrates on the basic concepts and discusses coherence, the interaction of radiation and matter, the Fabry-Perot interferometer, and oscillation conditions and methods of excitation of maser systems. The desirable spectroscopic characteristics for solid-state materials for maser application in the optical region are listed and details of the properties of crystals in use are described, together with references to the various possible applications of optical masers to physical and mathematical problems. The review, though somewhat demanding of the reader in physical and mathematical knowledge, is an excellent and comprehensive survey of the fundamental principles and of the progress of development up to September 1962. The second review, by W. R. Bennett, Yale University, is more limited in scope since he deals only with gaseous optical masers. There are four main sections. The first contains a summary of the basic considerations and the second of the dominant excitation mechanisms which have been used to produce population inversions in gas lasers. The third and main section deals with specific gaseous optical masers, in particular the helium-neon optical maser, the cæsium maser, neonoxygen and argon-oxygen masers and pure noble gas

* Optical Masers: Applied Optics. Supplement No. 1. Pp. 142. (Washington, D.C.: Optical Society of America. 1962.) 5 dollars. masers. Finally, in the fourth section, spectral characteristics and mode-pulling effects are discussed.

Solid optical masers require crystal rods with ends polished very flat and accurately parallel, and with the ends perpendicular to prism or eylinder axes. The special techniques involved and the methods used in the fabrication of such rods are briefly but clearly described in the article by W. L. Bond. Two articles deal with specific applications of ruby optical masers-the one to high-speed photography by T. Yajima, F. Shimizu and K. Shimoda, and the other as a source for the excitation of Raman spectra, by S. P. S. Porto and W. L. Wood.

In their original discussion of masers in $1958 \mathrm{M}$. Schawlow and C. H. Townes suggested the use of a FabryPerot interferometer as a multi-mode cavity and such an arrangement has since been utilized extensively. The general properties of this device when the emitting or absorbing atoms are put between the reflecting mirrors are described by A. Kastler. His paper is in French. $\mathrm{He}$ calculates the distribution of light intensity inside the interferometer and shows that the local light intensity in the stationary waves inside the cavity can be much higher than the intensity of the incident light beam. The light emitted by the atoms and emerging from the interferometer is investigated and it is shown that narrow fringes of very strong intensity can be obtained. It is emphasized that these properties are not only of application to lasers, but are also of importance in metrology and in spectroscopy.

Threshold conditions of masers for higher frequencies are discussed by K. Shimoda; population inversion in $a$ discharge in a mixture of two gases by N. G. Basov and O. M. Krokhin (this paper is printed in English and Russian in parallel columns); the coherence time of a maser, by H. E. J. Neugebauer; and non-linear optical effects, by A. E. Siegman. Scattered throughout the Supplement there is a large number of most interesting photographs of experimental optical maser set-ups, in addition to numerous references to papers on or relating to optical masers.

S. WEINTROUB

\section{CONTROL OF HYDATID DISEASE}

TTTLE is heard in most countries about movements Lo prevent the tapoworm of dogs, Echinococcus granulosus, from causing hydatid disease in human beings. The importance of this disease varies geographically in a curious manner. In most countries it is almost ignored, even when the danger is real, but in some countries it is given some attention, and in Australasia it is given a lot of attention. In New Zealand there is a National Hydatids Council, which recently published its second annual report*. * National Hydatids Council. Second Annual Report and Statement of
Accounts for the Year ended March 31, 1962. Pp. 32. (Wellington, N.Z.: Accounts for the Year end 